\title{
APPLICATION OF CONFORMAL MAPPING TO VISCOUS FLOW BETWEEN MOVING CIRCULAR CYLINDERS*
}

BY

LEE A. SEGEL

National Physical Laboratory, Teddington, England

\begin{abstract}
This work shows that conformal mapping provides an effective way to solve certain unsteady two-dimensional perturbation problems involving the flow of a viscous incompressible fluid, in particular flow between moving circular cylinders. If the outer cylinder is considered fixed, the principal motions treated are the slow rotation of a slightly eccentric inner cylinder, and the vibration of an inner cylinder about a slightly eccentric point. Mapping the given circular boundaries (of a crosssection) into concentric circles enables one to solve for the stream function by means of a series.

In the first problem, the solution is carried far enough to afford an estimate for convergence. The torque on the inner cylinder and the second order steady streaming are computed, and a necessary re-examination of the meaning of the second concept is given. Various special cases of the second problem are shown to illuminate the concepts of virtual mass and viscous damping. The results for high frequency vibration bear on an experimental paper by Stuart and Woodgate, some of the low frequency results on theoretical papers by Andres and Ingard, and by Stokes. Both problems are of general interest in connection with the design of certain control mechanisms, and with experiments on Helium II.
\end{abstract}

1. Introduction. Successful applications of conformal mapping to problems of fluid mechanics go back over a hundred years [3] and continue to the present. (See for example the many papers of L. C. Woods in the Proceedings of the Royal Society (A) and elsewhere.) Almost all of these applications have concerned two-dimensional flows of an inviscid incompressible fluid, since the well-known result

$$
\begin{gathered}
\frac{\partial^{2} \Psi(x, y)}{\partial x^{2}}+\frac{\partial^{2} \Psi(x, y)}{\partial y^{2}}=\left[\frac{\partial^{2} \psi(\xi, \eta)}{\partial \xi^{2}}+\frac{\partial^{2} \psi(\xi, \eta)}{\partial \eta^{2}}\right]\left|\frac{d \xi}{d z}\right|^{2} \\
\Psi[x(\xi, \eta), y(\xi, \eta)] \equiv \psi(\xi, \eta)
\end{gathered}
$$

concerning the transformation of the Laplace operator under a conformal transformation

$$
z=z(\zeta) \quad(z=x+i y, \zeta=\xi+i \eta)
$$

means that the governing (Laplace) equation for the stream function remains invariant if the plane of the independent variables is subjected to a conformal transformation. Consequently, any awkward boundary shape can be changed into a more convenient one, the only penalty being (generally) an alteration in the conditions which the stream function must satisfy on that boundary.

The object of this paper is to show that the use of conformal mapping provides an effective way to solve certain two-dimensional problems involving the flow of a

*Received October 19, 1959. 
viscous incompressible fluid. The governing equation for $\Psi$ is now more complicated(2.8) with $J^{*}=1$-and is no longer invariant under a conformal transformation, but if the awkward boundary is near a convenient one then the use of conformal mapping to exchange the former boundary for the latter will cause only a small alteration in the equation and boundary conditions, so that the transformed problem can be handled by standard perturbation methods. The particular problems discussed here involve flows forced by small amplitude motions of a boundary and therefore demonstrate the effectiveness of this approach in what might be termed "time dependent boundaryperturbation problems."

That conformal mapping would provide a useful method of attacking boundary perturbation problems was suggested several years ago by Lin [5]. In another paper [10], Segel has proved that the method is a valid one for such problems involving the equation $\partial^{2} w / \partial x^{2}+\partial^{2} w / \partial y^{2}+\lambda w=0$ ( $\lambda$ a constant), e.g., for determining the possible modes and frequencies of vibrating membranes bounded by an ellipse of small eccentricity ("near-circle") or by a "near" isosceles right triangle. The present paper and [10] therefore comprise a detailed investigation, practical and theoretical, of Lin's suggestion.

The particular flow problems selected as examples are sufficiently complicated both to furnish a good indication of the scope and limitations of the method and to provide illustration and clarification of such general concepts of fluid mechanics as virtual mass and steady streaming. Furthermore, two-dimensional flows between moving nearly concentric circular cylinders currently are of great interest in connection with the design of control mechanisms for aircraft and rockets, and with experiments on Helium II [14].

2. Rotating cylinders: formulation. Consider two right circular cylinders of radii $r_{1}$ and $r_{2}, r_{2}>r_{1}$. Supposing that their respective axes are always parallel, we investigate the two-dimensional motion caused when the axis of the inner cylinder rotates in a circular path about the axis of the outer. Let the complex $z$-plane represent a crosssection perpendicular to the axes. The intersection of the plane with the cylinders is two circles; let $z_{1}=r_{1} \in \exp [i \omega \alpha(t)]$ and $z_{2}=0$ be the position of their centers at time $t$. Here $1 / \omega$ is some typical time associated with the motion, $\epsilon$ is a dimensionless constant, and $\alpha$ is any real valued function of the time $t$.

As is well known, the bilinear mapping $\zeta=(A z+B) /(C z+D)$ sends "circles" into "circles". (A "circle" is a circle or a straight line.) It would seem likely that proper choice of the constants $A, B, C$, and $D$ would enable one to map the given eccentric $z$-plane configuration into concentric circles in the $\zeta$-plane. This is indeed possible: if $\zeta=\rho \exp (i \phi)$, the mapping

$$
\zeta=\frac{z+r_{1}(\delta-\epsilon) \exp (i \omega \alpha)}{\delta z+(1-\delta \epsilon) r_{1} \exp (i \omega \alpha)}
$$

with

$$
\delta=-2 \epsilon\left\{\left(r_{2}^{2} / r_{1}^{2}\right)-1-\epsilon^{2}+\left[\left\{\left(r_{2}^{2} / r_{1}^{2}\right)-1-\epsilon^{2}\right\}^{2}-4 \epsilon^{2}\right]^{1 / 2}\right\}^{-1},
$$

sends the moving circle $\left|z-z_{1}\right|=r_{1}$ and the fixed circle $|z|=r_{2}$ into the fixed concentric circles $\rho=1$ and $\rho=\beta$. (Compare [4].) Here

$$
\beta=\left(r_{2} / r_{1}\right)(1-\epsilon \delta)^{-1} \text {. }
$$

Instead of employing $r_{2} / r_{1}$ and $\epsilon$, the actual dimensionless geometric parameters 
of the problem, we will express the solution in terms of $\beta$ and $\delta$. To this end we note that

$$
\epsilon=-\left(\beta^{2}-1\right) \delta /\left(1-\beta^{2} \delta^{2}\right) .
$$

In order to obtain the equations of motion in the $\zeta$ plane, we observe that if $u$ and $v$ are the velocities in the direction of $\rho$ increasing and of $\phi$ increasing respectively, then the continuity equation is $[2, \mathrm{p} .114]$

$$
\partial\left(\rho J^{1 / 2} u\right) / \partial \rho+\partial\left(J^{1 / 2} v\right) / \partial \phi=0,
$$

where the Jacobian $J$ and the dimensionless Jacobian $J^{*}$ are given by

$$
J=r_{1}^{2} J^{*}=\left|\frac{d z}{d \zeta}\right|^{2}=\frac{\partial(x, y)}{\partial(\xi, \eta)}=\frac{r_{1}^{2}\left(1-\delta^{2}\right)^{2}}{\left(1-2 \delta \rho \cos \phi+\rho^{2} \delta^{2}\right)^{2}} .
$$

If we therefore define a dimensionless stream function $\psi$ by

$$
u=r_{1}^{2} \omega \rho^{-1} J^{-1 / 2}(\partial \psi / \partial \phi), \quad v=-r_{1}^{2} \omega J^{-1 / 2}(\partial \psi / \partial \rho),
$$

the continuity equation is automatically satisfied. The equation of motion is now easily found. Writing the $z$-plane Navier-Stokes equation in vorticity form (i.e. eliminating the pressure) and using (1.1) and the related result

$$
\frac{\partial\left(\Psi_{1}, \Psi_{2}\right)}{\partial(x, y)}=\frac{\partial\left(\psi_{1}, \psi_{2}\right)}{\partial(\xi, \eta)} \frac{\partial(\xi, \eta)}{\partial(x, y)},
$$

we arrive immediately at the equation of motion

$$
\frac{\partial(\Delta \psi)}{\partial t}+\frac{1}{\rho} \frac{\partial\left(\Delta \psi / J^{*}, \psi\right)}{\partial(\rho, \phi)}=\frac{1}{R} \Delta\left(\Delta \psi / J^{*}\right),
$$

where $\Delta \psi=\left(\partial^{2} \psi / \partial \rho^{2}\right)+\rho^{-1}(\partial \psi / \partial \rho)+\rho^{-2}\left(\partial^{2} \psi^{\prime} \partial \phi^{2}\right), R$ is the Reynolds number

$$
R=r_{1}^{2} \omega \cdot \nu \text {, }
$$

and $\nu$ is the kinematic viscosity.

Before establishing boundary conditions, we must specify the physical problem under consideration more precisely. For as the inner cylinder rotates as a whole about the origin, it may retain its orientation with respect to the line joining $z_{1}$ and $z_{2}$, or with respect to the outer circle, or it may rotate about its own center in some other prescribed manner. We consider here the case in which the inner circle retains its orientation with respect to the fixed outer circle. The other constant rotition cases require only a simple modification of the boundary conditions.

The case in which both $z_{1}$ and $z_{2}$ are fixed, $z_{1} \not \neq z_{2}$, and $\left|z_{1}-z_{2}\right|$ is small, and in which each circle rotates about its own center with angular velocities $\omega_{1}$ and $\omega_{2}$, is treated by Wood [15] who uses the time independent version of $(2.1)$ appropriate to his problem, writing it down as a change of variables with no comment. He then expands the stream function and boundary conditions in powers of $\delta$. Essentially the same problem as Wood's was also treated by Nikitin [8]. The latter used polar coordinates and therefore had to expand the position of the boundary, as well as the stream function and the boundary conditions, in powers of the appropriate small parameter.

In order to formulate our boundary conditions, we suppose, with no loss of generality, that $\alpha(0)=0$. Then if at $t=0$ a point on the inner circle has position $z(0)=r_{1} \epsilon+$ 
$r_{1} \exp (i \theta)$, at time $t$ the point will be at $z(t)=r_{1} \epsilon \exp (i \omega \alpha(t))+r_{1} \exp (i \theta)$ and will have velocity $z^{\prime}(t)=i r_{1} \epsilon \omega \alpha^{\prime}(t) \exp (i \omega \alpha(t))$ with components $\alpha^{\prime} \omega r_{1} \epsilon \sin (\theta-\omega \alpha)$ and $\alpha^{\prime} \omega r_{1} \epsilon \cos (\theta-\omega \alpha)$ respectively normal and tangent to the inner circle. But, from (2.1), when $z=r_{1} \epsilon \exp (i \omega \alpha)+r_{1} \exp (i \theta)$,

$$
\zeta=\exp (i \phi)=\{\exp [i(\theta-\omega \alpha)]+\delta\} /\{\delta \exp [i(\theta-\omega \alpha)]+1\}
$$

With this we can easily find $\cos (\theta-\omega \alpha)$ and $\sin (\theta-\omega \alpha)$ in terms of $\phi$ :

$$
\cos (\theta-\omega \alpha)=\frac{\cos \phi-2 \delta+\delta^{2} \cos \phi}{1-2 \delta \cos \phi+\delta^{2}}, \quad \sin (\theta-\omega \alpha)=\frac{\sin \phi\left(1-\delta^{2}\right)}{1-2 \delta \cos \phi+\delta^{2}} .
$$

The boundary conditions on $\psi$ are therefore

$$
\text { at } \rho=1: \quad \begin{array}{ll}
\frac{\partial \psi}{\partial \phi} & =-\frac{\alpha^{\prime}\left(\beta^{2}-1\right) \delta\left(1-\delta^{2}\right)^{2} \sin \phi}{\left(1-\beta^{2} \delta^{2}\right)\left(1-2 \delta \cos \phi+\delta^{2}\right)^{2}}, \\
& \frac{\partial \psi}{\partial \rho}=\frac{\alpha^{\prime}\left(\beta^{2}-1\right) \delta\left(1-\delta^{2}\right)\left(\cos \phi-2 \delta+\delta^{2} \cos \phi\right)}{\left(1-\beta^{2} \delta^{2}\right)\left(1-2 \delta \cos \phi+\delta^{2}\right)^{2}} .
\end{array}
$$

at $\rho=\beta: \quad \partial \psi / \partial \phi=\partial \psi / \partial \rho=0$.

As will be examined in more detail later, the boundary conditions (2.11) and (2.12) must be supplemented by the condition that the pressure be single-valued. Then the parabolic differential equation (2.8), the boundary conditions (2.11) and (2.12), and an initial condition of the form

$$
t=0: \psi(\rho, \phi, 0) \text { prescribed, }
$$

together comprise a well-defined mathematical problem.

The problem is non-linear. In order to be able to solve it, we restrict ourselves to the case in which the distance $\left|z_{1}-z_{2}\right|$ between the centers is small: that is, to the case in which $\epsilon$, and hence $\delta$, is small. In the standard way, we assume that, for sufficiently small $\delta, \psi$ is given by the series

$$
\psi=\psi_{1} \delta+\psi_{2} \delta^{2}+\cdots
$$

and we expand the differential equation and boundary conditions in powers of $\delta$, set the coefficients of $\delta, \delta^{2}, \cdots$ equal to zero, and obtain

$$
\begin{aligned}
& 0(\delta): \Delta \Delta \psi_{1}-R \partial\left(\Delta \psi_{1}\right) / \partial t=0 . \\
& \rho=1: \partial \psi_{1} / \partial \rho=\alpha^{\prime}\left(\beta^{2}-1\right) \cos \phi, \quad \partial \psi_{1} / \partial \phi=-\alpha^{\prime}\left(\beta^{2}-1\right) \sin \phi . \\
& \rho=\beta: \partial \psi_{1} / \partial \rho=\partial \psi_{1} / \partial \phi=0 \text {. } \\
& 0\left(\delta^{2}\right): \Delta \Delta \psi_{2}-R \partial\left(\Delta \psi_{2}\right) / \partial t=\frac{R}{\rho} \frac{\partial\left(\psi_{1}, \Delta \psi_{1}\right)}{\partial(\rho, \phi)}+4 \Delta\left(\rho \cos \phi \Delta \psi_{1}\right) \text {. } \\
& \rho=1: \partial \psi_{2} / \partial \rho=2\left(\beta^{2}-1\right) \cos 2 \phi, \quad \partial \psi_{2} / \partial \phi=-2\left(\beta^{2}-1\right) \sin 2 \phi . \\
& \rho=\beta: \partial \psi_{2} / \partial \rho=\partial \psi_{2} / \partial \phi=0 \text {. } \\
& (2.16 \mathrm{a}, \mathrm{b}, \mathrm{c}) \\
& 0\left(\delta^{3}\right): \Delta \Delta \psi_{3}-R \frac{\partial\left(\Delta \psi_{3}\right)}{\partial t}=\frac{R}{\rho}\left[\frac{\partial\left(\psi_{1}, \Delta \psi_{2}\right)}{\partial(\rho, \phi)}+\frac{\partial\left(\psi_{2}, \Delta \psi_{1}\right)}{\partial(\rho, \phi)}+\frac{\partial\left(\psi_{1},-4 \rho \cos \phi \Delta \psi_{1}\right)}{\partial(\rho, \phi)}\right] \\
& +\Delta\left[4 \rho \cos \phi \Delta \psi_{2}-2\left(1+\rho^{2}+2 \rho^{2} \cos ^{2} \phi\right) \Delta \psi_{1}\right] \\
& \rho=1: \partial \psi_{3} / \partial \rho=\left(\beta^{2}-1\right)\left(\beta^{2}-4+6 \cos 2 \phi\right) \text {, } \\
& \partial \psi_{3} / \partial \phi=-\left(\beta^{2}-1\right)\left(\beta^{2}+2+6 \cos 2 \phi\right) . \\
& \rho=\beta: \partial \psi_{3} / \partial \rho=\partial \psi_{3} / \partial \phi=0 \text {. etc. } \\
& (2.17 \mathrm{a}, \mathrm{b}, \mathrm{c})
\end{aligned}
$$


Once $\psi$ is found, the velocities $u$ and $v$ follow at once from (2.7), and the pressure $p(\rho, \phi)=\delta p_{1}+\delta^{2} p_{2}+\cdots$ can be found from the Navier-Stokes equations in $(\rho, \phi)$ coordinates (cf. [2, p. 101-103]). Since we will need it later, we note that $p_{1}$ satisfies

$$
\left.\rho_{0}^{-1}\left(\partial p_{1} / \partial \rho\right)=\nu \omega \rho^{-1}\left[\partial\left(\Delta \psi_{1}\right) \partial \phi\right)\right], \quad \rho_{0}^{-1}\left(\partial p_{1} / \partial \phi\right)=-\nu \omega \rho\left[\partial\left(\Delta \psi_{1}\right) / \partial \rho\right] .
$$

A formula for an important physical quantity is that for the counterclockwise torque about the origin which it is necessary to exert in order to keep the inner cylinder rotating in the prescribed manner. To derive it, we consider the moment caused by the stresses $P_{\rho \rho}$ and $P_{\rho \phi}$ acting on a given element $\Delta \phi$ of the inner circle. With the aid of a simple diagram and a little trigonometry, the counterclockwise moment exerted by the fluid on the element is seen to be

$$
\left[P_{\rho \rho} r_{1} \epsilon \sin (\theta-\omega \alpha)+P_{\rho \phi} r_{1}(1+\epsilon \cos (\theta-\omega \alpha)] J^{1 / 2} \Delta \phi .\right.
$$

The factor $J^{1 / 2}$ must be included since $P_{\rho \rho}$ and $P_{\rho \phi}$ are actual stresses; to find the force they exert on $\rho=1$ one must therefore multiply by the length element $|d z|=$ $|d z / d \zeta||d \zeta|=J^{1 / 2} d \phi$. To obtain the desired torque $T$, we change the sign and integrate

$$
\left.T=-r_{1} \int_{0}^{2 \pi}\left\{P_{\rho \rho} \epsilon \sin (\theta-\omega \alpha)+P_{\rho \phi}[1+\epsilon \cos (\theta-\omega \alpha))\right]\right\} J^{1 / 2} d \phi .
$$

$P_{\rho \rho}$ and $P_{\rho \phi}$ can be written in terms of $u$ and $v$, and hence $\psi$, by [2, p. 103] so that the coefficients $T$, in the expansion $T=T_{1} \delta+T_{2} \delta^{2}+\cdots$ can be found using (2.10) and (2.4).

3. Constant angular velocity. We consider now the special (and most interesting) case when the inner circle rotates about the origin with constant angular velocity $\omega$. Here $\alpha(t)=t, \alpha^{\prime}(t)=1$. The boundary conditions are time independent, so that $\psi$ is as well. The equation for $\psi_{1}$ is then $\Delta \Delta \psi_{1}=0$ so, from the boundiry conditions, the form of $\psi_{1}$ is evidently

$$
\psi_{1}=\left(\beta^{2}-1\right) \cos \phi c(\rho) .
$$

Since $e(\rho)$ satisfies an equi-dimensional ordinary differential efulution, it is easily found to be

$$
e(\rho)=C_{1} \rho^{-1}+C_{2} \rho \ln \rho+C_{3} \rho+C_{4} \rho^{3} .
$$

From the boundary conditions $(2.15 \mathrm{~b}, \mathrm{c})$, with $\alpha^{\prime}=1$ of course, we find

$$
C_{1}=-\frac{\beta^{2}}{2 d_{1}}, C_{2}=\frac{-\left(\beta^{2}+1\right)}{d_{1}}, C_{3}=\frac{2 \beta^{2} \ln \beta+2 \ln \beta-\beta^{2}+1}{2 d_{1}}, C_{4}=\frac{1}{2 d_{1}},
$$

where

$$
d_{1}=\beta^{2} \ln \beta+\ln \beta-\beta^{2}+1 .
$$

But the solution just found is not the only one satisfying $\Delta \Delta \psi_{1}=0$ and the boundary conditions $(2.15 \mathrm{~b}, \mathrm{c})$. In fact

$$
h(\rho)=b_{1}+b_{2} \ln \rho+b_{3} \rho^{2}+b_{4} \rho^{2} \ln \rho
$$

satisfies $\Delta \Delta h=0$, so provided only that the two requirements $h^{\prime}(1)=0$ and $h^{\prime}(\beta)=0$ are satisfied, $\left(\beta^{2}-1\right)(e)(\cos \phi)+h$ satisfies the same equation and boundary con- 


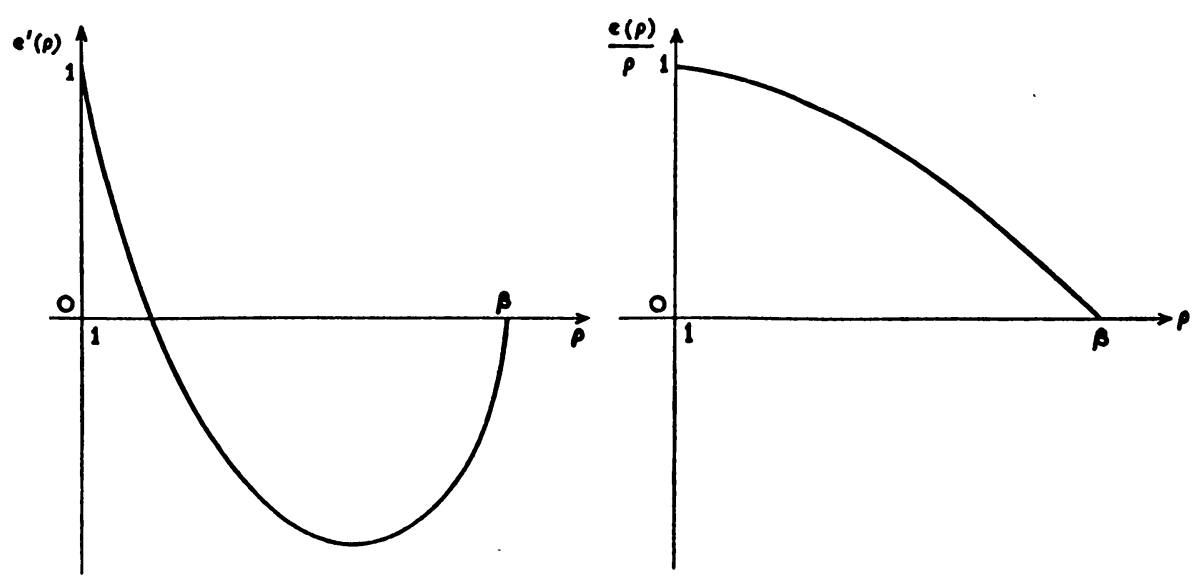

Fig. 1. Shapes of $e(\rho) / \rho$ and $e^{\prime}(\rho)$.

ditions as $\left(\beta^{2}-1\right)(\cos \phi)(e)$ itself: the four arbitrary constants in $h$ are not determined by the two above requirements on $h^{\prime}$. Recall however that the pressure must be singlevalued. Since $\partial(\Delta h) / \partial \rho=4 b_{4} \rho^{-1}$, we see from $(2.18 \mathrm{~b})$ that unless $b_{4}=0, p_{1}$ will have a term proportional to $\phi$ and will not be single-valued. Hence $b_{4}=0$, the requirements on $h^{\prime}$ imply that $b_{2}=b_{3}=0$, and the stream function is determined up to an arbitrary additive constant.

The solution obtained is the only time independent one to the $O(\delta)$ equations (2.15) with $\alpha^{\prime}=1$. However, any time dependent solution to (2.15a), having vanishing space derivatives on $\rho=1$ and $\rho=\beta$, could still be added to the $\psi_{1}$ of (3.1), and appropriate initial conditions could be satisfied by superposition of these solutions. The result would represent a flow forced by the assigned initial conditions and by the revolving cylinder. We are interested only in the motion caused by the revolving cylinder; consequently the $\psi_{1}$ of (3.1) is, as it stands, the desired first order stream function.

Before considering the higher approximations, let us examine some of the features of the flow as given by the first order solution. We have performed calculations for $\beta=2.718$ corresponding to a configuration in which the outer radius is about three times the inner. The particular value of $\beta$ was of course chosen in order that $\ln \beta=1$, thereby simplifying the calculations.

With $\beta=2.718$, the functions $e(\rho) / \rho$ and $e^{\prime}(\rho)$ have the shapes given in Fig. 1 . Since the first order approximations to the velocities are $u_{1} \delta=\left(\rho^{-1} \partial \psi_{1} / \partial \phi\right) \delta=$ $(-\delta)\left(\beta^{2}-1\right) \rho^{-1} e \sin \theta$ and $v_{1} \delta=\left(-\partial \psi_{1} / \partial \rho\right) \delta=(-\delta)\left(\beta^{2}-1\right) e^{\prime} \cos \phi$, the "flow" in the $\zeta$ plane forms two symmetric vortices. At a given instant, the flow in the physical plane will therefore have the slightly asymmetric appearance of Fig. 2.

Since the inverse of (2.1) is

$$
z=r_{1} \exp (i \omega t) \frac{(1-\epsilon \delta)+\epsilon-\delta}{1-\delta \zeta} \equiv F(\zeta) \exp (i \omega t)
$$

the velocity field looks steady to an observer rotating with angular velocity $\omega$. (This also follows at once from the fact that an observer sitting on the inner cylinder would see a fixed geometrical configuration in which moves a fluid driven by the steady counterclockwise rotation of the outer cylinder.) The fluid is pushed away from the front of 


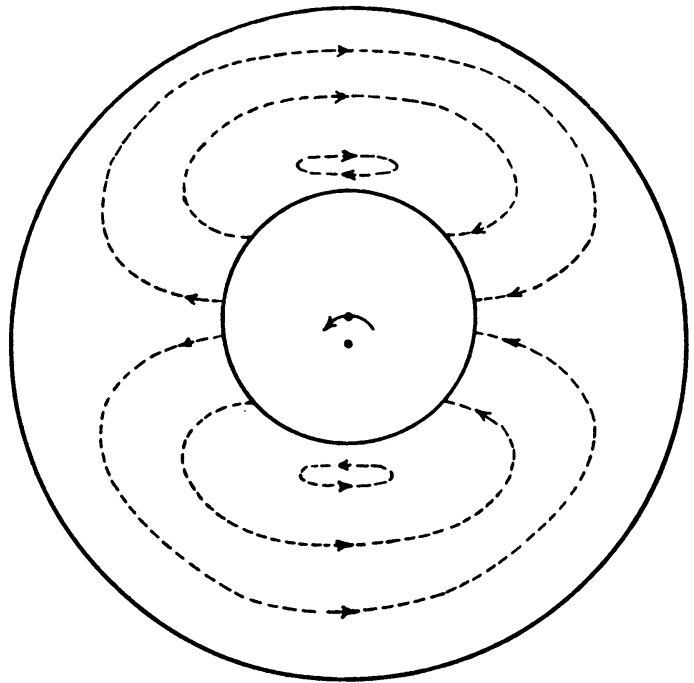

FIG. 2. Rotating inner cylinder: instantaneous streamlines.

the inner cylinder and then is deflected outward and backward by the outer cylinder. It flows inward and forward again at the back, filling the gap left by the retreating inner cylinder.

We must explain briefly why we have spoken of "flows" in the $\zeta$ plane. If one uses conformal mapping for determining the flow of inviscid incompressible fluids, it is certainly permissible to speak of a flow in the transformed plane, since the governing equation (Laplace's) is invariant. Here, the governing equation is changed so that the "flow" in the transformed plane is not generally speaking a possible flow of a real fluid.

If $\delta$ is small enough to insure convergence of the $\psi$ series, the flow should not depart appreciably from the first order flow described above. Nonetheless, it is necessary to calculate higher approximations in order to find how small $\delta$ need be-and to compute such quantities as the torque $T$, for (3.1) can be shown to give $T_{1}=0$. (This can also be deduced from symmetry arguments which demonstrate that $T$ must be an even function of $\delta$.)

Therefore we note that with the value of $\psi_{1}$ already computed, the $O\left(\delta^{2}\right)$ equation (2.16a) becomes

$$
\begin{aligned}
\Delta \Delta \psi_{2}=R\left(\beta^{2}-1\right)^{2} \sin 2 \phi\left[C_{2}^{2}\left(\frac{2 \ln \rho+1}{\rho^{2}}\right)\right. & \left.+\frac{2 C_{2} C_{3}-8 C_{1} C_{4}}{\rho^{2}}+8 C_{4}^{2} \rho^{2}\right] \\
& +\left(\beta^{2}-1\right)\left(64 C_{4}-16 C_{2} \rho^{-2} \cos 2 \phi\right),
\end{aligned}
$$

so that its solution is of the form

$$
\psi_{2}=\left(\beta^{2}-1\right)[f(\rho) \cos 2 \phi+g(\rho) \sin 2 \phi+h(\rho)] .
$$

Substitution of (3.8) into (3.7) and solution of the resulting differential equations subject to the boundary conditions $(2.16 \mathrm{~b}, \mathrm{c})$ show that

$$
f(\rho)=A_{1} \rho^{-2}+A_{2}+A_{3} \rho^{2}+A_{4} \rho^{4}+C_{2} \rho^{2} \ln \rho,
$$




$$
\begin{aligned}
g(\rho)=B_{1} \rho^{-2} & +B_{2}+B_{3} \rho^{2}+B_{4} \rho^{4} \\
& +R\left(\beta^{2}-1\right)\left[\frac{C_{4}^{2} \rho^{6}}{48}+\frac{C_{2} C_{4}}{6} \rho^{4} \ln \rho\right. \\
& \left.+\frac{16 C_{1} C_{4}-C_{2}^{2}-4 C_{2} C_{3}}{32} \rho^{2} \ln \rho-\frac{C_{2}^{2}}{16} \rho^{2} \ln ^{2} \rho\right],
\end{aligned}
$$

$h(\rho)=D_{1}+D_{2} \ln \rho+D_{3} \rho^{2}+D_{4} \rho^{2} \ln \rho+C_{4} \rho^{4}$.

By an argument parallel to the one around (3.5), it can be shown that $p_{2}$ is single valued if and only if $D_{4}=0$. Computation of the correct values for the remaining constants is now straightforward but tedious. The results are given in [9] as are the complicated $\psi_{3}$ equation and its lengthy solution. With them one can derive the torque formula

$$
T_{2} / \nu \rho_{0} \omega r_{1}^{2}=\left(\beta^{2}-1\right)\left[2 C_{1}+C_{2}+2 C_{4}\left(4 \beta^{2}-1\right)-\left(\beta^{2}-1\right)\left(\frac{1}{2}+C_{1}+\frac{3}{2} C_{2}-C_{4}\right)\right] .
$$

Also, since the convergence radius of the assumed expansions in powers of $\delta$ cannot be estimated rigorously, the magnitudes of the higher order corrections to $\psi$ must be used to give a "practical" estimate. All we know a priori is that we have neglected the nonlinear convection terms, as opposed to the diffusion terms, in (2.8) so that we must have $\delta^{2}$ "much less" than $\delta / R$. Although we can choose $R$ to be as large as we like and then choose $\delta$ to make $\delta \ll 1 / R$, we have assumed that diffusion is the dominant process in the flow so that our approximation is essentially a low Reynold's number one.

Calculations of the tangential velocity $v$ [9] for $\beta=2.718$ and $R=1$ show that if $\delta=-0.01$, the third order correction to $v$ is at most $21 / 2$ per cent of the second approximation so that in this case "convergence for $|\delta|$ much less than $1 / R$ " means "convergence for $|\delta|$ less than about $10^{-2} / R$ ". If one used for a fluid "Rotring" motor oil $\left(\nu=9 \mathrm{~cm}^{2} \mathrm{sec}^{-1}, \mu=8 \mathrm{gm} \mathrm{cm}^{-1} \mathrm{sec}^{-1}\right.$ at 1 atmosphere and $20^{\circ} \mathrm{C}$ according to p. 230 of Schlichting's Grenzschicht-Theorie) the case $\beta=2.718, R=1, \delta=-0.01$ might correspond to an inner cylinder of radius $1.2 \mathrm{~cm}$ rotating once per second within an outer cylinder of radius $3.2 \mathrm{~cm}$, the axis of the inner cylinder being displaced $0.08 \mathrm{~cm}$ from the axis of the outer. This displacement is 6.4 per cent of the inner radius and 4 per cent of the outer. The value of the torque per unit length in this case is $6.7 \times 10^{-2}$ dyne-cm/cm.

Many details of the flow are most easily determined by obtaining the required quantity from $\psi(\rho, \phi)$ evaluated at the point $(\rho, \phi)$ corresponding to the point $(r, \theta)$ in question: the torque calculation was carried out in this manner. In some instances, however, it is necessary to find $\Psi(r, \theta)$ explicitly by developing from an expansion of (2.1) series such as

$$
\begin{gathered}
\rho=s+\delta\left(\beta^{2}-s^{2}\right) \cos (\theta-\omega t)+\cdots \quad\left(s=r / r_{1}\right), \\
\cos \phi=\cos (\theta-\omega t)+\delta(1 / 2) s^{-1}\left(\beta^{2}+s^{2}\right)[1-\cos 2(\theta-\omega t)]+\cdots,
\end{gathered}
$$

and thereby computing $\Psi(r, \theta)=\Psi_{0}(r, \theta)+\delta \Psi_{1}(r, \theta)+\cdots$ from $\psi(\rho, \phi)$.

The most important quantities to be found from $\Psi(r, \theta)$-or, more correctly, from $\Psi(r, \theta, t)$-are the steady streaming velocities $u_{s}(r, \theta)$ and $v_{s}(r, \theta)$. Since a computation shows that

$$
\begin{aligned}
\Psi_{s} & \equiv T^{-1} \int_{0}^{T} \Psi(r, \theta, t) d t=\delta^{2}\left[\frac{\beta^{2}+s^{2}}{2 s} e(s)+\frac{\beta^{2}-s^{2}}{2} e^{\prime}(s)+h(s)\right]+O\left(\delta^{3}\right) \\
& \equiv \delta^{2} \Psi_{2 s}+O\left(\delta^{3}\right) \quad(T=2 \pi / \omega)
\end{aligned}
$$


the leading contributions to the streaming are $u_{s}=0+O\left(\delta^{3}\right)$ and $v_{s} /\left(r_{1} \omega\right)=-\partial \Psi_{2 s} / \partial r=$ $-\left(C_{2}+4 C_{4}\right)\left(\beta^{2} s^{-1}-s\right)$ giving (to this order) a steady tangential flow opposite to the direction of rotation, its magnitude increasing from zero at the outer cylinder $(s=\beta)$ to $-\left(C_{2}+4 C_{4}\right)\left(\beta^{2}-1\right)$ at the inner cylinder $(s=1)$. The fact that the direction of the streaming is opposite to what one would expect seems to be common to low Reynold's number problems. (See Sec. 6.)

It is at first alarming that although the boundary conditions require that the tangential velocity at a point $(r, \theta)$ of the inner cylinder be proportional to $\cos (\theta-\omega t)$ and therefore have a zero time average, our calculation shows a non-zero streaming at the inner boundary. To resolve this difficulty, it is necessary to examine more closely the meaning of these calculations. What we have done is to compute the average velocity at a given point $(r, \theta)$ from a coordinate system fixed in the stationary outer cylinder; the results could be verified experimentally by suspending an appropriate device at $(r, \theta)$ from a frame fixed to the outer cylinder. Viewed either mathematically or experimentally, it is clear that this procedure can have no meaning for points inside $r=$ $r_{1}(1+\epsilon)$, for every such point is for a time inside the rotating inner cylinder. During this time, the velocity at such points is undefined so a formal mathematical average has no meaning, and obviously an instrument, even if it could pass through the cylinder, would produce irrelevant readings.

If one were standing on the inner cylinder, no difficulties in measuring the streaming would be apparent near it, but the same difficulties would appear near the outer. The theoretical result here is $u_{s}=0, v_{s}=r_{1} \omega\left(C_{2}+4 C_{4}\right)(s-1)$, again giving a tangential streaming opposite to the direction of rotation seen by the observer. To sum up, in a flow caused by boundaries in motion relative to each other, there is a different steady streaming with respect to each of the boundaries; to single out this aspect of the flow generally has no meaning near to boundaries other than that one selected for reference.

4. Variable angular velocity. If the inner cylinder rotates with a variable angular velocity, i.e. if $\alpha$ is no longer considered to be a linear function of $t$, we write the first order equation (2.15) as

$$
\partial Q_{1} / \partial t=R^{-1} \Delta Q_{1}, \quad Q_{1}=\Delta \psi_{1} .
$$

If we assume separable solutions of the form

$$
Q_{1}=m(\rho)\left(\beta^{2}-1\right) \cos \phi \exp (i p t), \quad \psi_{1}=n(\rho)\left(\beta^{2}-1\right) \cos \phi \exp (i p t),
$$

then equations (4.1) are satisfied, for any constant $p$, if and only if

$$
\begin{gathered}
n^{\prime \prime}+\rho^{-1} n^{\prime}-\rho^{-2} n=m, \\
\rho^{2} m^{\prime \prime}+\rho m^{\prime}+\left(k^{2} \rho^{2}-1\right) m=0, \quad k^{2}=-i p R .
\end{gathered}
$$

Since (4.4) is a Bessel equation

$$
m(\rho)=A J_{1}(k \rho)+B Y_{1}(k \rho) ;
$$

$\mathrm{n}(\rho)$ can now be obtained by variation of parameters. In general, $n(\rho)$ will contain two more arbitrary constants (in addition to $A$ and $B$ ); in this case satisfaction of the two boundary conditions (2.15c) at $\rho=\beta$ requires these constants to be zero. Since $p$ is arbitrary, we superpose the solutions already obtained and attempt a solution of the form

$$
\psi_{1}=\left(\beta^{2}-1\right) \cos \phi \int_{-\infty}^{\infty} \exp (i p t)\left[\rho F(\rho, k)-\rho^{-1} G(\rho, k)\right] d p
$$


in which $k=(-i p R)^{1 / 2}$,

$$
\begin{aligned}
& F(\rho, k)=\int_{\rho}^{\beta}\left[A(k) J_{1}(k \xi)+B(k) Y_{1}(k \xi)\right] d \xi, \\
& G(\rho, k)=\int_{\rho}^{\beta}\left[A(k) J_{1}(k \xi)+B(k) Y_{1}(k \xi)\right] \xi^{2} d \xi,
\end{aligned}
$$

or, since the integrals in (4.7) and (4.8) can be evaluated, where

$$
\begin{array}{r}
F(\rho, k)=-k^{-1} A(k)\left[J_{0}(k \beta)-J_{0}(k \rho)\right]-k^{-1} B(k)\left[Y_{0}(k \beta)-Y_{0}(k \rho)\right], \\
G(\rho, k)=k^{-1} A(k)\left[\beta^{2} J_{2}(k \beta)-\rho^{2} J_{2}(k \rho)\right]+k^{-1} B(k)\left[\beta^{2} Y_{2}(k \beta)-\rho^{2} Y_{2}(k \rho)\right] .
\end{array}
$$

From the two remaining boundary conditions we therefore find that

$$
A(k)=\frac{k F(k)}{D(k)}\left[Y_{2}(k)-\beta^{2} Y_{2}(\beta k)\right], \quad B(k)=-\frac{k F(k)}{D(k)}\left[J_{2}(k)-\beta^{2} J_{2}(\beta k)\right],
$$

in which

$$
\begin{aligned}
D(k)=\left[J_{0}(k)-J_{0}(\beta k)\right]\left[Y_{2}(k)-\beta^{2} Y_{2}(\beta k)\right] & \\
& -\left[J_{2}(k)-\beta^{2} J_{2}(\beta k)\right]\left[Y_{0}(k)-Y_{0}(\beta k)\right] .
\end{aligned}
$$

With all this, we see finally that $\psi_{1}$ can be written as

$$
\psi_{1}=(2 \pi)^{-1 / 2}\left(\beta^{2}-1\right) \cos \phi \int_{-\infty}^{\infty} e^{i p t}\left[\frac{\rho Z_{1}(\rho, k)-\rho^{-1} Z_{2}(\rho, k)}{D(k)}\right] H(p) d p
$$

where we have made the abbreviations

$$
\begin{aligned}
Z_{1}(\rho, k)= & {\left[J_{2}(k)-\beta^{2} J_{2}(\beta k)\right]\left[Y_{0}(\beta k)-Y_{0}(\rho k)\right] } \\
& -\left[J_{0}(\beta k)-J_{0}(\rho k)\right]\left[Y_{2}(k)-\beta^{2} Y_{2}(\beta k)\right], \\
Z_{2}(\rho, k)=\left[Y_{2}(k)-\beta^{2} Y_{2}(\beta k)\right]\left[\beta^{2} J_{2}(\beta k)-\right. & \left.\rho^{2} J_{2}(\rho k)\right] \\
& -\left[\beta^{2} Y_{2}(\beta k)-\rho^{2} Y_{2}(\rho k)\right]\left[J_{2}(k)-\beta^{2} J_{2}(\beta k)\right],
\end{aligned}
$$

and where $H$ is the inverse Fourier transform of $\alpha^{\prime}$ :

$$
\alpha^{\cdot}(t)=(2 \pi)^{-1 / 2} \int_{-\infty}^{\infty} e^{-i p t} H(p) d p .
$$

(4.13) may be partially checked by noting that it should reduce to (3.1) when $\alpha^{\prime}(t)=1$. But when $\alpha^{\prime}=1,(2 \pi)^{-1 / 2} H=\delta(s)$, the Dirac delta function, and evaluation of (4.13) is afforded by finding the limit of $Z_{1}(\rho, k) / D(k)$ and $Z_{2}(\rho, k) / D(k)$ as $k \rightarrow 0^{+}$. This is most easily accomplished directly from the series definitions of the various Bessel functions involved; the computations show that (4.13) does reduce to (3.1) when $\alpha^{\prime}=1$.

Finally, behavior of $\psi_{1}$ at large time can sometimes be found at once from known asymptotic formulas for Fourier transforms. Using a formula in ([7], p. 483), since for $p \rightarrow 0$ the square bracket in the integrand of (4.13) behares like

if

$$
\frac{1}{2} \frac{\left(\beta^{2}-\rho^{2}\right)\left(3 \rho-\rho^{-1}\right)-\left(\beta^{2}+1\right) \ln (\beta / \rho)}{\beta^{2}-1-\left(\beta^{2}+1\right) \ln \beta}+O(p),
$$

$$
H_{+}(p) \sim C p^{m} \text { for } p \rightarrow 0 \quad \text { where } H_{+}(p)=0 \text { for } p<0 \text { ) }
$$


then as $t \rightarrow \infty$

$$
\psi_{1} \sim\left(\beta^{2}-1\right) \cos \phi \frac{C \Gamma(m+1)}{t^{m+1}} \frac{(1 / 2)\left(\beta^{2}-\rho^{2}\right)\left(3 \rho-\rho^{-1}\right)-\left(\beta^{2}+1\right) \ln (\beta / \rho)}{\beta^{2}-1-\left(\beta^{2}+1\right) \ln \beta} .
$$

The $0(p)$ term in (4.15) has been evaluated, but it seems too complicated to be of interest; note, however, that terms of $0(p \ln p)$, which arise from the Bessel functions of the second kind $Y_{0}$ and $Y_{2}$, cancel, so that the remainder in (4.15) is $0(p)$.

5. Vibrating cylinders: formulation and first approximation. Let us again consider the two circular cylinders of Sec. 2, only this time let the center of the inner cylinder remain fixed at $z=z_{1}=0$ while $z_{2}$, the center of the outer, vibrates about $r_{1} \epsilon$ according to $z_{2}=r_{1} \epsilon(1+\cos \omega t)$. Neither cylinder will be allowed to rotate about its center, though, as before, such rotations present no difficulty aside from more complicated computations. This problem is a natural complement to that of Sec. 2; it also contains different features which may be associated with the fact that there is no longer a coordinate system in which the problem is steady.

To solve, we proceed as before. The conformal mapping

$$
\zeta=\left(z-r_{1} \delta\right) /\left(r_{1}-\delta z\right)
$$

transforms the circle with center $z_{1}$ and radius $r_{1}$ into $\rho=1$, and the circle with center $z_{2}$ and radius $r_{2}$ into the concentric circle $\rho=\beta$. Note that (with the abbreviation $\alpha=$ $\left.r_{2} / r_{1}\right)$ the value of $\delta$,

$$
\delta=-\frac{2 \epsilon(1+\cos \omega t)}{\alpha^{2}-1-\epsilon^{2}(1+\cos \omega t)^{2}+\left\{\left[\alpha^{2}-1-\epsilon^{2}(1+\cos \omega t)^{2}\right]^{2}-4 \epsilon^{2}(1+\cos \omega t)^{2}\right\}^{1 / 2}}
$$

varies with time so that the mapping (5.1), and the Jacobian

$$
J=|d z / d \zeta|^{2}=r_{1}^{2}\left(1-\delta^{2}\right)^{2}\left(1+2 \rho \delta \cos \phi+\delta^{2} \rho^{2}\right)^{-2}
$$

are now functions of time. The dimensionless stream function (defined as in (2.7)) will therefore satisfy

$$
J \frac{\partial}{\partial \tau}\left(\frac{\Delta \psi}{J}\right)+\frac{1}{\rho} \frac{\partial(\Delta \psi / J, \psi)}{\partial(\rho, \phi)}=\frac{1}{R} \Delta(\Delta \psi / J) \quad(\tau=\omega t)
$$

with appropriate boundary conditions on $\rho=1$ and $\rho=\beta$. The outer circle, while now concentric with the inner, has a time varying radius

$$
\beta=\alpha[1-\delta \epsilon(1+\cos \tau)]^{-1} .
$$

To make the boundaries into fixed concentric circles, one would have either to make a further radial (non-conformal) transformation or to use the standard series expansion method, discussed in [10]. However, as

$$
\beta=\alpha+\epsilon^{2}(1+\cos \tau)^{2} \alpha\left(\alpha^{2}-1\right)^{-1}+\cdots=\alpha\left[1+O\left(\epsilon^{2}\right)\right],
$$

the effect of the variation of $\beta$ with time need not be taken into account until the third approximation is considered.

Taking $\alpha$ and $\epsilon$ as the geometric parameters of the problem, we assume that $\psi=$ $\psi_{1}+\psi_{2} \epsilon^{2}+\cdots$ and proceed as usual. Noting that

$$
-\delta=\epsilon(1+\cos \tau)\left(\alpha^{2}-1\right)^{-1}+2 \epsilon^{3}(1+\cos \tau)^{3}\left(\alpha^{2}-1\right)^{-2}+\cdots,
$$

we find that (5.4) separates into

$$
O(\epsilon): R \partial\left(\Delta \psi_{1}\right) / \partial \tau-\Delta \Delta \psi_{1}=0 .
$$


$O\left(\epsilon^{2}\right): R \partial\left(\Delta \psi_{2}\right) / \partial \tau-\Delta \Delta \psi_{2}=-R_{\rho^{-1}} \partial\left(\Delta \psi_{1}, \psi_{1}\right) / \partial(\rho, \phi)+\frac{4 R \rho \cos \phi \sin \tau \Delta \psi_{1}}{\alpha^{2}-1}$

while the boundary conditions are

$$
+\Delta\left[+\rho \cos \phi(1+\cos \tau) \Delta \psi_{1}\right]\left(\alpha^{2}-1\right)^{-1},
$$

$$
\begin{aligned}
O(\epsilon): \rho & =1: \partial \psi_{1} / \partial \rho=\partial \psi_{1} / \partial \phi=0 . \\
\rho & =\alpha: \partial \psi_{1} / \partial \rho=-\sin \phi \sin \tau, \quad \partial \psi_{1} / \partial \phi=-\alpha \cos \phi \sin \tau . \\
O\left(\epsilon^{2}\right): \rho & =1: \partial \psi_{2} / \partial \rho=\partial \psi_{2} / \partial \phi=0 . \\
\rho & =\alpha: \partial \psi_{2} / \partial \rho=-\alpha\left(\alpha^{2}-1\right)^{-1}(2 \sin \tau+\sin 2 \tau) \sin 2 \phi . \\
& \partial \psi_{2} / \partial \phi=-\alpha^{2}\left(\alpha^{2}-1\right)^{-1}(2 \sin \tau+\sin 2 \tau) \cos 2 \phi .
\end{aligned}
$$

As (5.8) is the same as (4.1), we follow the procedure of Sec. 4 to obtain the following solution to (5.8) and (5.10):

where, if $S=i^{3 / 2} R^{1 / 2}$

$$
\psi_{1}=\operatorname{Im}[e(\rho) \sin \phi \exp (i \tau)]
$$

$$
\begin{array}{r}
e(\rho)=-(A / 2 S)\left\{\rho\left[J_{0}(S \rho)-J_{0}(S)\right]+\rho^{-1}\left[\rho^{2} J_{2}(S \rho)-J_{2}(S)\right]\right\} \\
-(B / 2 S)\left\{\rho\left[Y_{0}(S \rho)-Y_{0}(S)\right]+\rho^{-1}\left[\rho^{2} Y_{2}(S \rho)-Y_{2}(S)\right]\right\}, \\
\begin{aligned}
A=2 S\left[\alpha^{2} Y_{2}(S \alpha)-Y_{2}(S)\right] / d(\alpha), \quad B=-2 S\left[\alpha^{2} J_{2}(S \alpha)-J_{2}(S)\right] / d(\alpha), \\
d(\alpha)=\left[J_{0}(S \alpha)-J_{0}(S)\right]\left[\alpha^{2} Y_{2}(S \alpha)-Y_{2}(S)\right] \\
-\left[\alpha^{2} J_{2}(S \alpha)-J_{2}(S)\right]\left[Y_{0}(S \alpha)-Y_{0}(S)\right] .
\end{aligned}
\end{array}
$$

The most interesting physical quantity to be derived from this result is $F_{H}$, the horizontal force on the inner cylinder caused by the prescribed motion of the outer. (The vertical force is zero by symmetry.) A computation shows that the force (taken positive in the direction of $x$ increasing) on a length $L$ of the cylinder is

$$
F_{H}=\operatorname{Im}\left\{R^{-1} M U_{0} \omega \exp (i \omega t) S\left[A J_{2}(S)+B Y_{2}(S)\right][1+O(\epsilon)]\right\},
$$

where, for reasons discussed below, we have made the abbreviations

$$
U_{0}=r_{1} \epsilon \omega, \quad M=\rho_{0} \pi r_{1}^{2} L .
$$

The problem (call it $P$ ) under consideration is closely related to the problem $P^{\prime}$ of determining the motion caused if the inner cylinder vibrates $\left(z_{1}=-r_{1} \epsilon(1+\cos \omega t)\right)$ while the outer remains stationary $\left(z_{2}=0\right)$. The connection between the two is seen by the following standard argument. The problem $P$ originally discussed is governed by

$$
\partial u / \partial t+u \partial u / \partial x+v \partial u / \partial y=-\rho_{0}^{-1} \partial p / \partial x+\nu\left(\partial^{2} u / \partial x^{2}+\partial^{2} u / \partial y^{2}\right),
$$

the corresponding equation for $v$, the continuity equation, and the boundary conditions appropriate to the vibration of the outer cylinder. (For this paragraph only, $u$ and $v$ represent velocities in the $x$ and $y$ directions.) If we make the change of variables

$x^{\prime}=x-\epsilon r_{1}(1+\cos \omega t), \quad t^{\prime}=t, \quad y^{\prime}=y, \quad u^{\prime}=u+r_{1} \epsilon \omega \sin \omega t$,

and

$$
p^{\prime}=p-\rho_{0} r_{1} \epsilon \omega^{2} \cos \omega t \quad x=p-\rho_{0} r_{1} \epsilon \omega^{2} \cos \omega t \quad r \cos \theta
$$


we get a new problem with equations identical with (5.18) etc. but, due to the change from $x$ to $x^{\prime}$, with boundary conditions appropriate to problem $P^{\prime}$. Consequently a solution to $P^{\prime}$ can be related to a solution of $P$ by (5.19) and (5.20). In particular, the pressure on the inner cylinder for $P^{\prime}$ is found from the corresponding pressure for $P$ by subtracting $\rho_{0} r_{1} \epsilon \omega^{2} \cos \omega t \cos \theta$. This means that the force on the inner cylinder in $P^{\prime}$ is found from (5.16) by adding

$$
\Delta^{\prime}=M U_{0} \omega \cos \omega t
$$

Until now, the only assumption we have made is that $|\epsilon| \ll 1$, i.e. that the amplitude of vibration is small compared with the radius of the inner cylinder. As is seen from (5.4), this allows us to neglect convection terms (to a first approximation) regardless of the magnitude of $R$. That is (5.12) and (5.16) give the first order solution for small amplitude vibrations, regardless of their frequency. As these expressions are complicated, it is however advisable to examine their behavior for high and low frequencies.

For large $R$ (and hence large $|S|), \psi_{1}=\operatorname{Im}\left[e_{\infty}(\rho) \sin \phi \exp (i \tau)\right]$ where, using the asymptotic formulas for $J$ and $Y$

$$
\begin{aligned}
e_{\infty}(\rho)=-A_{\infty}\left(\frac{2}{\pi S}\right)^{1 / 2}\left[\frac{2}{S} \rho^{-1 / 2} \sin \left(S-\frac{\pi}{4}\right)\right. & -\left(\rho-\frac{1}{\rho}\right) \cos \left(S-\frac{\pi}{4}\right) \\
& \left.-\frac{1}{8 S}\left(\rho+\frac{15}{\rho}\right) \sin \left(S-\frac{\pi}{4}\right)\right] \\
-B_{\infty}\left(\frac{2}{\pi S}\right)^{1 / 2}\left[\frac{2}{S} \rho^{-1 / 2} \cos \left(S \rho-\frac{\pi}{4}\right)\right. & +\left(\rho-\frac{1}{\rho}\right) \sin \left(S-\frac{\pi}{4}\right) \\
& \left.-\frac{1}{8 S}\left(\rho+\frac{15}{\rho}\right) \cos \left(S-\frac{\pi}{4}\right)\right], \\
A_{\infty}= & d_{\infty}^{-1}(2 / \pi S)^{1 / 2}\left[\sin (S-\pi / 4)-\alpha^{3 / 2} \sin (S \alpha-\pi / 4)\right], \\
B_{\infty}= & d_{\infty}^{-1}(2 / \pi S)^{1 / 2}\left[\cos (S-\pi / 4)-\alpha^{3 / 2} \cos (S \alpha-\pi / 4)\right], \\
d_{\infty}= & (2 / \pi S) \alpha^{-1 / 2}\left(\alpha^{2}-1\right) \sin [S(\alpha-1)] .
\end{aligned}
$$

It is necessary to include the "small" $O(1 / S)$ terms in the square brackets of (5.22) because the "order one" term is in fact zero at the inner boundary. The region in which the $O(1 /|S|)$ terms are important is given approximately by $\left(\rho-\rho^{-1}\right) \leq|1 / S|$ or $\rho \leq 1+R^{-1 / 2}, r=r_{1} \rho \leq 1+(\nu / \omega)^{1 / 2}$. As the term proportional to $\left(\rho-\rho^{-1}\right)$ is the inviscid solution, we see that for high frequency oscillations the influence of viscosity is primarily confined to a boundary layer near the inner cylinder having thickness of order $(\nu / \omega)^{1 / 2}$.

Using (5.22), one can compute the asymptotic form of the force on the inner cylinder due to high frequency oscillations of the outer. The result is

$$
F_{H \omega}=\alpha^{2}\left(\alpha^{2}-1\right)^{-1} M U_{0} \omega\left\{\left[2+2(2 / R)^{1 / 2}\right] \cos \omega t+2(2 / R)^{1 / 2} \sin \omega t\right\} .
$$

From (5.21), when the inner cylinder is oscillating with high frequency as described in Problem $P^{\prime}$, the force exerted by the cylinder (i.e. the force required to move it) is then

$-F_{H \infty}^{\prime}=\alpha^{2}\left(\alpha^{2}-1\right)^{-1} M U_{0} \omega\left\{\left[\alpha^{-2}\left(\alpha^{2}+1\right)+2(2 / R)^{1 / 2}\right] \cos \omega t+2(2 / R)^{1 / 2} \sin \omega t\right\}$. 
Since the center of the inner cylinder is at $r_{1} \epsilon(1+\cos \omega t)$, it has velocity $V=r_{1} \epsilon \omega \sin$ $\omega t=U_{0} \sin \omega t$. Consequently, if a length $L$ of the inner cylinder had mass $M^{*}$ it would require a force of $M^{*} \omega U_{0} \cos \omega t$ to move it in the absence of a fluid. The fluid's presence causes the appearance of an additional force $M U_{0} \omega\left(\alpha^{2}-1\right)^{-1}\left[\alpha^{2}+1+2 \alpha^{2}(2 / R)^{1 / 2}\right]$ in phase with the acceleration, so the cylinder behaves as if it had the additional "virtual mass" $M\left(\alpha^{2}-1\right)^{-1}\left[\alpha^{2}+1+2 \alpha^{2}(2 / R)^{1 / 2}\right]$. The dependence of this expression on $\alpha$ reflects the influence of the fixed outer cylinder on the force required to move the inner. If the outer cylinder is made to recede indefinitely $(\alpha \rightarrow \infty)$ the virtual mass approaches $M\left[1+2(2 / R)^{1 / 2}\right.$, which is Stokes' result for a vibrating circular cylinder in an infinite fluid [12]. As $R \rightarrow \infty$ the virtual mass approaches $M$, the mass of fluid displaced; as it should be, this is the inviscid result.

The concept of virtual mass arises from the fact that a body moving in an inviscid fluid is subject to an extra inertia force which can be found by augmenting the body's mass by a multiple of the mass of fluid it displaces, the numerical value of the multiple depending on the nature of the motion and the shape of the body. If the fluid is slightly viscous, the inviscid solution still holds, approximately, except for the boundary layer region of slow motion near the body, so that one expects to find a correction to the virtual mass due to the increase in the apparent size of the body caused by the boundary layer. (We assume that separation does not occur.) In the problem considered here, the viscous correction term to the virtual mass, $\left[2 \alpha^{2}(2 M)^{1 / 2}\right] /\left[\left(\alpha^{2}-1\right) R^{1 / 2}\right]$, may be interpreted in this way by writing it as $2^{1 / 2} A \rho_{0} L \alpha^{2} /\left(\alpha^{2}-1\right)$ since $A=2 \pi r_{1}(\nu / \omega)^{1 / 2}$ is of the order of the area effectively added to the inner cylinder by the boundary layer.

In addition to the virtual mass force, there is a viscous damping force

$$
-2(2 / R)^{1 / 2} \alpha^{2}\left(\alpha^{2}-1\right)^{-1} M U_{0} \omega \sin \omega t
$$

on the body, opposing its motion. The coefficient here approaches $2 \cdot 2^{1 / 2}=2.83$ as $\alpha \rightarrow \infty$. In experiments by Martin [6] and by Stuart and Woodgate [13], the values 2.87 and 2.94 were found for this constant. The small discrepancies could be due to any of several factors, but as some finite container for the fluid surrounding the vibrating cylinder must have been used, it is worth noting that a bounding outer cylinder of 8.5 and 5 times the radius of the inner could have produced the observed discrepancies. Stuart in fact recalls that the ratio $\alpha$ for their experiment was of the order of 10 (the outer container was cylindrical) so that about one-fourth of the observed discrepancy is attributable to the presence of the outer cylinder. Martin used a vibrating wire in a complicated set up: a photograph of his apparatus (in his paper) indicates that the nearest obstacle is so many diameters away from the wire that the effect discussed here is insignificant in his experiment.

For small $R$, the first order stream function is approximately

$$
\begin{gathered}
\psi_{1}=\left(2 d_{0}\right)^{-1} \sin \phi \sin \omega t\left[\rho^{3}+\left(\alpha^{2}-1\right) \rho-\alpha^{2} \rho^{-1}-2\left(\alpha^{2}+1\right) \rho \ln \rho\right], \\
d_{0}=\left(\alpha^{2}+1\right) \ln \alpha-\alpha^{2}+1,
\end{gathered}
$$

so that the response of the fluid is very nearly in phase with the forcing movement of the boundary. The leading term in expression (5.16) for the force on the inner cylinder due to vibration of the outer is

$$
F_{H O}=-4\left(\alpha^{2}+1\right)\left(d_{0} R\right)^{-1} M U_{\mathrm{\nu}} \omega \sin \omega t ;
$$


since $\Delta^{\prime}$ of (5.21) is independent of $R$, this is also the leading term in the expression for the force on the inner cylinder in problem $P^{\prime}$. The principal force in low Reynolds number vibration is therefore a viscous damping which becomes infinite as $R$ approaches zero.

Since $d\left[\left(\alpha^{2}+1\right) / d_{0}\right] / d \alpha=-\left(\alpha^{2}-1\right)^{2} \alpha^{-1} d_{0}^{-2}<0$, the viscous damping decreases from an infinite value as the ratio of the radii increases from unity (at fixed $R$ ). Note however that (5.28) is valid only for finite $\alpha$ as it was derived under the assumptions that $|S|$ and $|\alpha S|$ are small. That is, mathematically, a small value of $|\alpha S|$ was assumed in obtaining simplified expressions for $A$ and $B$ in (5.16) so that in discussing the variation of (5.28) with $\alpha$, we assume that $R$ is fixed, and so small that (5.28) is valid for a range of $\alpha$. The solution for small Reynolds number oscillations in an unbounded fluid can only be obtained by first letting $\alpha$ tend to infinity and then taking the limit for small $R$. (See Stokes' results described below.) Physically, if $|S|=R^{1 / 2}=r_{1} /(\nu / \omega)^{1 / 2}$ is small, then the thickness of the boundary layer is large compared to the radius of the inner cylinder: if $|\alpha S|=r_{2} /(\nu / \omega)^{1 / 2}$ is also small, then the thickness of the boundary layer is also large compared to the radius of the outer cylinder. Consequently, in deriving (5.28) we have assumed that viscosity is important in the entire region between the two cylinders.

Taking a further term in the expansion of (5.16) for small $|\alpha S|$, we find that the first correction to (5.28) is a force

$$
-\frac{\alpha^{6}+3 \alpha^{4}-6 \alpha^{4} \ln \alpha-3 \alpha^{2}-6 \alpha^{2} \ln \alpha-1}{6 d_{0}^{2}} M U_{0} \omega \cos \omega t=-C_{0}(\alpha) M U_{0} \cos \omega t
$$

acting on the inner cylinder due to the vibration of the outer. This means that for small $R$ the virtual mass coefficient for problem $P^{\prime}$ is very nearly $C_{0}(\alpha)-1$. Examination of (5.29) shows that $C_{0}(\alpha)$ is infinite for $\alpha=1$ and for $\alpha=\infty$, and therefore has a minimum for some intermediate $\alpha$.

As in [12], Stokes' result for the force on a cylinder oscillating at small $R$ in an unbounded fluid can be found from the general formula of linear theory

$$
\begin{aligned}
F_{H}=\operatorname{Im} & \left\{-i M U_{0} \omega e^{i \omega t}\left[1-\frac{4 K_{1}\left[(i R)^{1 / 2}\right]}{K_{1}\left[(i R)^{1 / 2}\right]+i R K_{1}^{\prime}\left[(i R)^{1 / 2}\right]}\right]\right\} \\
& =\operatorname{Im}\left\{-i M U_{0} \omega e^{i \omega t}\left[K-i K^{\prime}\right]\right\}=-M U_{0} \omega\left[K \cos \omega t+K^{\prime} \sin \omega t\right],
\end{aligned}
$$

where $K_{1}$ is the modified Bessel function of the second kind as defined in [16], p. 374]. For small $R, K$ and $K^{\prime}$ reduce to

$$
\begin{aligned}
& K=1+\pi / R\left(L^{2}+\pi^{2} / 16\right), \quad K^{\prime}=4 L / R\left(L^{2}+\pi^{2} / 16\right), \\
& L=\ln (4 / R)^{1 / 2}-\gamma, \gamma=\text { Euler's constant }=0.577 \cdots,
\end{aligned}
$$

which are the expressions obtained by Stokes [11]. If the oscillating cylinder is enclosed by another cylinder, our work gives the results

$$
K=C_{0}(\alpha)-1, \quad K^{\prime}=4\left(\alpha^{2}+1\right) /\left(d_{0} R\right) .
$$

A comparison of (5.31) and (5.32) shows that as $R \rightarrow 0$ the damping coefficient becomes infinite like $1 /(R \ln R)$ for a cylinder oscillating in an unbounded fluid, and like $1 / R$ if the fluid is bounded externally, within the boundary layer, by another cylinder. 
In the first case, the virtual mass coefficient also becomes infinite, more slowly, like $1 /\left(R \ln ^{2} R\right)$ while in the second, the virtual mass coefficient remains finite.

It is possible to find suggestive physical interpretations for the derived variation of the force with $R$ and with $\alpha$. Let us refer to the Stokes problem and the problem considered in this paper as the "unbounded" and "bounded" problems respectively, and let us consider the force on the inner cylinder due to its own vibration. That for fixed $\alpha$, in both cases, this force increases without limit as the Reynolds number tends to zero can be regarded simply as reflecting the fact that vibrations in a very viscous fluid are heavily damped.

That as $R \rightarrow 0$, the virtual mass tends to infinity in the unbounded problem but approaches a constant in the bounded problem can be illuminated as follows. In the solution to the unbounded problem, the radial factor in the stream function is a linear combination of $1 / r$ and $K_{1}\left[r(i R)^{1 / 2}\right]$ [12]. $K_{1}$ arises because of the inclusion of the $\partial \psi / \partial t$ term and, mathematically, allows one to satisfy the viscous no-slip boundary conditions at the cylinder. Physically, as in [11], if Stokes' solution is regarded as giving the behavior at large time of a fluid agitated by a circular cylinder which at some instant begins a sinusoidal oscillation from rest, then the $\partial \psi / \partial t$ term can be seen to provide the inertia needed to curb what would otherwise be a continual increase in the amount of fluid carried by the cylinder as it persisted in its oscillations. (Similarly, if a fluid with constant velocity $U$ at infinity flows past a cylinder, this curbing is accomplished by non-linear terms like $u \partial u / \partial x$-linearly approximated by Oseen as $U \partial u / \partial x$.) Since if $R$ is very small, the velocities arising from $K_{1}$ are important until $r$ becomes very large, it is clear that at small $R$ the influence of viscosity extends far into the fluid from the cylinder. The fact that the virtual mass in the unbounded problem tends to infinity as $R \rightarrow 0$ is thus seen to be due to the fact that the amount of fluid pushed and dragged by the cylinder-due to viscosity-also tends to infinity as $R \rightarrow 0$.

If the fluid is bounded externally, the situation is different. The radial coordinate is now bounded so that if $R$ is sufficiently small, the inertia terms are uniformly small compared to the terms retained. As we have seen, the leading contribution to the stream function is then the quasi-steady (5.26) which represents the in-phase response of an inertia-less fluid to the motion of its boundaries. The next most important term in the stream function gives rise to a finite virtual mass because the amount of fluid pushed and dragged is at most the finite amount contained between the two cylinders.

From a simple point of view, the variation of the force with $\alpha$ should be governed by the fact that the nearer the outer cylinder is to the inner, the greater should be the influence of the outer cylinder, i.e. the greater should be the force on the inner-which accounts for the decrease of the viscous damping with increasing $\alpha$ (at fixed small $R$ ). But although the virtual mass first decreases as $\alpha$ increases, it reaches a minimum and then starts increasing again. This is because the virtual mass is proportional to the mass times the acceleration of the fluid pushed and dragged by the inner cylinder. Now the closer the outer cylinder is to the inner, the faster $u$ and $v$ must change from the values imposed by the ribrating inner cylinder to the zero ralues imposed by the stationary outer cylinder, so that as $\alpha$ increases the derivatives of $u$ and $v$, i.e. the accelerations, decrease. But when $\alpha$ increases the amount of accelerated fluid increases. It appears that at small $\alpha$ the former process dominates, at large $\alpha$, the latter.

Once again, the above discussion of the qualitative effects of the outer cylinder is valid only if $|\alpha S|=\alpha R^{1 / 2}=\left(r_{2}^{2} \omega / \nu\right)^{1 / 2}$ is small. Thus, discussing the pendulum experi- 
ments of Bailey, Stokes [11, pp. 83-7] uses (5.31) in computing the correction to the virtual mass, and hence to the period, of the pendulum due to the thin wire by which the spherical pendulum was suspended. This is justified (and our calculations assuming small $|\alpha S|$ are not relevant) because although $|S|$ ranges from $10^{-1}$ to $10^{-2}$ for the various cases considered, and is certainly small, the outer cylinder is so far from the wire that $|\alpha S|$ is of the order $10^{2}$ and is thus not small but large. Appropriate simplifications of (5.16) for large $|\alpha S|$ and small $|S|$ (the case of a boundary layer thin compared with the radius of the outer cylinder but thick compared with the radius of the inner) could easily be worked out, but the effect of the outer cylinder on Stokes' already small corrections would be insensible.

6. Second approximation: small R. $\psi_{2}$ is found by solving the differential equation (5.9) under the boundary conditions (5.11); it clearly has the form

$$
\psi_{2}=\sin 2 \phi\left[C_{1}(\rho)+C_{2}(\rho) \sin \tau+C_{3}(\rho) \cos \tau+C_{4}(\rho) \sin 2 \tau+C_{5}(\rho) \cos 2 \tau\right] .
$$

Since the solutions of the homogeneous equations are known, the inhomogeneous equations for the C's may be solved by variation of parameters. Finding $\psi_{2}$ therefore presents no difficulties other than computational ones, but these are formidable due to the complicated expression obtained on substituting $\psi_{1}$ into the right hand side of (5.9), and due to the fact that two of the four solutions to the homogeneous equations are also complicated combinations of Bessel functions.

Interesting results are still forthcoming if we make the simplifying assumption that $R$ is small and expand the known function $\psi_{1}$ and the unknown function $\psi_{2}$ in powers of $R$

$$
\psi_{1}=\psi_{10}+R \psi_{11}+\cdots, \psi_{2}=\psi_{20}+R \psi_{21}+\cdots .
$$

We obtain from (5.9) and (5.11)

$$
\begin{gathered}
-\Delta \Delta \psi_{20}=\left(\alpha^{2}-1\right)^{-1} \Delta\left[4 \rho \cos \phi(1+\cos \tau) \Delta \psi_{10}\right], \\
\rho=1: \partial \psi_{20} / \partial \rho=\partial \psi_{20} / \partial \phi=0 . \\
\rho=\alpha: \partial \psi_{20} / \partial \rho=-\alpha\left(\alpha^{2}-1\right)^{-1}(2 \sin \tau+\sin 2 \tau) \sin 2 \phi, \\
\partial \psi_{20} / \partial \phi=-\alpha^{2}\left(\alpha^{2}-1\right)^{-1}(2 \sin \tau+\sin 2 \tau) \cos 2 \phi, \\
-\Delta \Delta \psi_{21}=-\frac{1}{\rho} \frac{\partial\left(\Delta \psi_{10}, \psi_{10}\right)}{\partial(\rho, \phi)}+\frac{\Delta\left[4 \rho \cos \phi(1+\cos \tau) \Delta \psi_{11}\right]}{\alpha^{2}-1} \\
+\frac{4 \rho \cos \phi \sin \tau}{\alpha^{2}-1} \Delta \psi_{10}-\frac{\partial\left(\Delta \psi_{20}\right)}{\partial \tau} . \\
\rho=1: \partial \psi_{21} / \partial \rho=\partial \psi_{21} / \partial \phi=0 . \quad \rho=\alpha: \partial \psi_{21} / \partial \rho=\partial \psi_{21} / \partial \phi=0
\end{gathered}
$$

from which we can obtain $\psi_{20}$ and $\psi_{21}$. On substitution of $\psi_{10}$ from (5.26), (6.3) becomes

$$
-\Delta \Delta \psi_{20}=-8\left(\alpha^{2}+1\right) d_{0}^{-1}\left(\alpha^{2}-1\right)^{-1}[2 \sin \tau+\sin 2 \tau] \rho^{-2} \sin 2 \phi .
$$

The solution of this equation and the boundary conditions (6.4) has the form

$$
\begin{gathered}
\psi_{20}=f(\rho) \sin 2 \phi[2 \sin r+\sin 2 \tau], \\
f(\rho)=A_{1} \rho^{-2}+A_{2}+A_{3} \rho^{2}+A_{4} \rho^{4}-\left(\alpha^{2}+1\right)\left[2\left(\alpha^{2}-1\right) d_{0}\right]^{-1} \rho^{2} \ln \rho,
\end{gathered}
$$




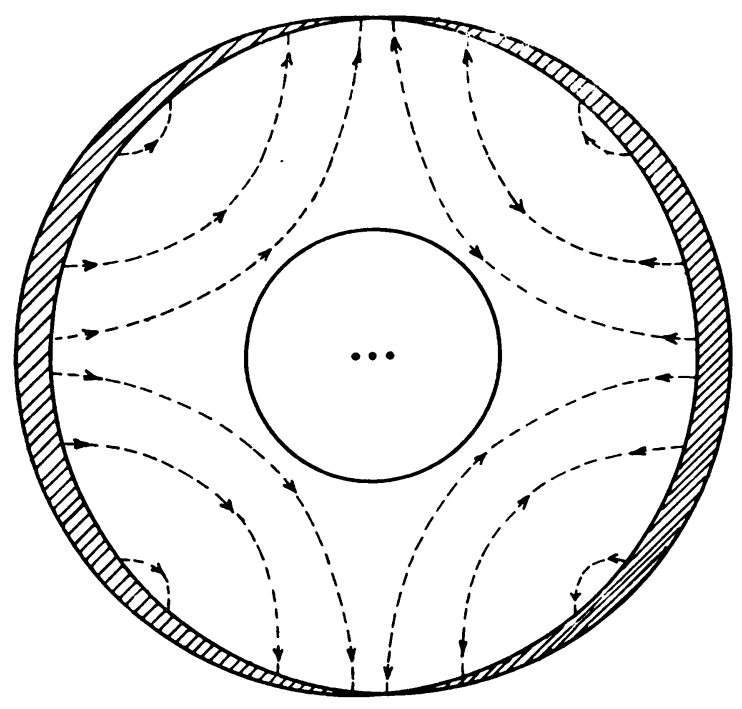

Fia. 3. Vibrating outer cylinder: steady streaming. (Since points in the hatched region are for a time outside the outer cylinder, a steady streaming velocity cannot be usefully defined in this region.)

so that the equation for $\psi_{21}$ can be derived and the solution found to be of the form

$$
\begin{aligned}
\psi_{21}=\frac{(\cos 2 \tau-1) \sin 2 \phi}{d_{0}^{2}} h_{1}(\rho)-\frac{1+2 \cos \tau+\cos 2 \tau}{\left(\alpha^{2}-1\right) d_{0}^{2}} & \sin 2 \phi h_{2}(\rho) \\
& \quad+8(\cos \tau+\cos 2 \tau) \sin 2 \phi h_{3}(\rho) .
\end{aligned}
$$

The details are given in [9].

We observe the appearance of a second order steady streaming with stream function

$$
\psi_{2,}=\frac{\epsilon^{2} R \sin 2 \phi}{\alpha^{2}-1}\left[\frac{1}{4}\left(\rho+\frac{1}{\rho}\right) e_{1}+\frac{1}{4}\left(\rho^{2}-1\right) e_{1}^{\prime}-\left(\alpha^{2}-1\right) h_{1}-h_{2}\right] \text {. }
$$

The first tivo terms arise from $\psi_{1}$ just as in the discussion of streaming at the end of Sec. 3. (We have expanded the $e(\rho)$ in (5.12) as $e(\rho)=e_{0}(\rho)-i R e_{1}(\rho)+O\left(R^{2}\right)$.) The last two terms arise from the aperiodic part of $\psi_{2}$ (not present in the case of Sec. 3).

A computation shows that for $\alpha=2.718$ the steady streaming forced by a slowly vibrating outer cylinder (Fig. 3) shows little qualitative difference from that forced by a stream slowly oscillating at infinity. (For the latter, see [1, Fig. 4].) One must beware of a (false) conception of steady streaming as some sort of actual flow; this would lead to the erroneous intuitive idea that the presence of the outer cylinder should cause the $\psi_{2 s}$ streamlines to bend around into closed paths. In fact, as discussed in Sec. 3, the steady streaming velocity is an artificially singled-out part of the actual velocity. Failure to realize that this singling-out has no meaning near the outer cylinder (to be precise, in the hatched region of Fig. 3) would lead to the incorrect ideas just mentioned.

Acknowledgement. Almost all of this work formed a portion of a Ph.D. thesis in the Mathematics Department, Massachusetts Institute of Technology. Part of it was done with support of a contract from the United States Office of Naval Research, and part at the National Physical Laboratory.

It is a pleasure to acknowledge the help and encouragement of Prof. C. C. Lin of 
M.I.T. Valuable suggestions were also received from Prof. L. Howard of M.I.T. and from Dr. J. T. Stuart and Mr. J. Watson of N.P.L.

\section{BiBLIOGRAPHY}

1. J. Andres and U. Ingard, Acoustic streaming at low Reynold's numbers, J. Acoust. Soc. Amer. 25, 932-8 (1953)

2. S. Goldstein, Ed., Modern developments in fluid dynamics, Clarendon Press, Oxford, 1938

3. G. Kirchoff, Pogg. Ann. 94 (1845), as in Ges. Abhandl. 1, Leipzig, 1882

4. H. Kober, Dictionary of conformal representation, Dover, N. Y., 1952

5. C. C. Lin, On a perturbation theory based on the method of characteristics, J. Math. Phys. 33, 132-3 (1954)

6. H. Martin, Über Tonhöhe und Dämpfung der Schwingungen von Saiten in verschiedenen Flüssigkeiten, Ann. Phys. (4) 77, 627-57 (1925)

7. P. Morse and H. Feshbach, Methods of theoretical physics, McGraw-Hill, N. Y., 1953

8. A. Nikitin, $O$ dviženii vyazkot żidkosti meždu šcipom $i$ podšipnikom, (On the flow of a viscous fluid between pin and bearing), In ženernyY Sbornik 23, 173-85 (1956)

9. L. A. Segel, Applications of conformal mapping to boundary perturbation problems, Ph.D. Thesis, M. I. T. Math. Dept., 1959

10. L. A. Segel, Application of conformal mapping to boundary perturbation problems for the membrane equation, to be published

11. G. Stokes, On the effect of the internal friction of fluids on the motion of pendulums, Math. and Phys. Papers 3, The University Press, Cambridge, 1901, pp. 38-54

12. J. T. Stuart, Chap. VII of Laminar boundary layers, to be published by the Clarendon Press, Oxford, $1960 / 61$

13. J. T. Stuart and L. Woodgate, Experimental determination of the aerodynamic damping on a vibrating circular cylinder, Phil. Mag. 46, 40-46 (1955)

14. W. Vinen, Detection of single quanta of circulation in rotating Helium II, Nature 181, 1524-5 (1958)

15. W. W. Wood, The asymptotic expansions at large Reynolds numbers for steady motion between noncoaxial rotating cylinders, J. Fluid Mechanics 3, 159-75 (1957)

16. E. Whittaker and G. Watson, Modern analysis, The University Press, Cambridge, 1952 